\title{
Glutathione and glutamate in schizophrenia: a 7T MRS study
}

\author{
Jyothika Kumar ${ }^{1}$ Elizabeth B. Liddle ${ }^{1} \cdot$ Carolina C. Fernandes $^{2} \cdot$ Lena Palaniyappan $^{3,4} \cdot$ Emma L. Hall $^{2}$. \\ Siân E. Robson ${ }^{5}$ Molly Simmonite ${ }^{6} \cdot$ Jan Fiesal $^{7} \cdot$ Mohammad Z. Katshu $^{1,8} \cdot$ Ayaz Qureshi $^{9} \cdot$ Michael Skelton $^{10}$. \\ Nikolaos G. Christodoulou ${ }^{1,8} \cdot$ Matthew J. Brookes $^{2} \cdot$ Peter G. Morris $^{2} \cdot$ Peter F. Liddle $^{1}$
}

Received: 7 May 2017 / Revised: 4 May 2018 / Accepted: 14 May 2018 / Published online: 22 June 2018

(c) The Author(s) 2018. This article is published with open access

\begin{abstract}
In schizophrenia, abnormal neural metabolite concentrations may arise from cortical damage following neuroinflammatory processes implicated in acute episodes. Inflammation is associated with increased glutamate, whereas the antioxidant glutathione may protect against inflammation-induced oxidative stress. We hypothesized that patients with stable schizophrenia would exhibit a reduction in glutathione, glutamate, and/or glutamine in the cerebral cortex, consistent with a post-inflammatory response, and that this reduction would be most marked in patients with "residual schizophrenia", in whom an early stage with positive psychotic symptoms has progressed to a late stage characterized by long-term negative symptoms and impairments. We recruited 28 patients with stable schizophrenia and 45 healthy participants matched for age, gender, and parental socio-economic status. We measured glutathione, glutamate and glutamine concentrations in the anterior cingulate cortex (ACC), left insula, and visual cortex using 7T proton magnetic resonance spectroscopy (MRS). Glutathione and glutamate were significantly correlated in all three voxels. Glutamine concentrations across the three voxels were significantly correlated with each other. Principal components analysis (PCA) produced three clear components: an ACC glutathione-glutamate component; an insula-visual glutathione-glutamate component; and a glutamine component. Patients with stable schizophrenia had significantly lower scores on the ACC glutathione-glutamate component, an effect almost entirely leveraged by the sub-group of patients with residual schizophrenia. All three metabolite concentration values in the ACC were significantly reduced in this group. These findings are consistent with the hypothesis that excitotoxicity during the acute phase of illness leads to reduced glutathione and glutamate in the residual phase of the illness.
\end{abstract}

Electronic supplementary material The online version of this article (https://doi.org/10.1038/s41380-018-0104-7) contains supplementary material, which is available to authorized users.

\section{Peter F. Liddle}

peter.liddle@nottingham.ac.uk

1 Division of Psychiatry and Applied Psychology, University of Nottingham, Nottingham, UK

2 Sir Peter Mansfield Imaging Centre, University of Nottingham, Nottingham, UK

3 Departments of Psychiatry, Medical Biophysics and Neuroscience, Western University, London, ON, Canada

4 Lawson Research, Brain and Mind \& Robarts Research Institutes, London, ON, Canada

\section{Introduction}

Schizophrenia is a serious, episodic, and persisting illness, with a characteristic time course in which acute episodes, characterized by positive psychotic symptoms such as delusions and hallucinations, are followed by a chronic phase in which negative symptoms and disabling cognitive

5 Healthcare Improvement Scotland, Gyle Square, Edinburgh, UK

6 Department of Psychology, University of Michigan, Ann Arbor, MI, USA

7 South Staffordshire and Shropshire Healthcare NHS Foundation Trust, Stafford, UK

8 Nottinghamshire Healthcare NHS Foundation Trust, Nottingham, UK

9 Greater Manchester West Mental Health NHS Foundation Trust, Manchester, UK

10 Derbyshire Healthcare NHS Foundation Trust, Derby, UK 
and social impairments tend to be prominent. There is evidence for three potentially related pathophysiological processes consistent with the characteristic time course of illness.

Firstly, abnormally high levels of presynaptic striatal dopamine occur in at least some patients, and are associated with positive symptoms [1]. Treatment with dopamine antagonists tends to alleviate these positive symptoms. Secondly, the brain's major excitatory neurotransmitter, glutamate, is implicated [2]. One proposal is that hypofunction of NMDA receptors (NMDAR) located on inhibitory GABA inter-neurons leads to reduced activity of these inter-neurons, resulting in increased pyramidal glutamatergic neurotransmission and the diverse symptoms occurring in acute psychosis [3]. However, sustained glutamatergic over-activity might lead to excitotoxic damage resulting in residual negative symptoms, cognitive dysfunction, and occupational and social impairment [4]. Glutamatergic abnormalities in schizophrenia have been found in brain tissue studies [5]; genetic association studies [6]; and studies investigating the role of glutamatergic agonists to combat NMDAR hypofunction and reduce symptoms [7].

Thirdly, and potentially closely related to the glutamatergic hypothesis, is the theory of oxidative stress [8]. Oxidative stress can result either from the excessive production of reactive oxygen species, which might be anticipated at times of glutamatergic over-activity, or from a reduction in oxidative defences, and can damage cell structures. Brain cells are particularly susceptible, owing to their low antioxidant defences and high oxidative metabolic activity. Glutathione, the brain's major intracellular antioxidant, thus has a vital role to play in maintaining brain cell health. Evidence for a role for oxidative stress in schizophrenia includes studies reporting reduced glutathione blood levels [9], reduced post-mortem glutathione concentrations [10,11], and genetic evidence implicating schizophrenia-related polymorphisms in genes involved in glutathione synthesis [12].

Animal studies indicate a close relationship between glutamate and glutathione. Persson et al. [13] showed that glutathione synthesis was directly related to microglial glutamate uptake and the release of glutamate metabolites. Barger et al. showed that depletion in glutathione levels due to oxidative stress is related to microglial glutamate release [14]. In a review of the role of glutathione in protection against neuronal death, Bains et al. [15] present evidence that glutamatergic transmission activates biochemical pathways that generate free radicals and/or lower defence against damage by free radicals. Conversely, free radicals can increase the concentration of glutamate in the synaptic cleft by the release of glutamate or blockade of its reuptake. In particular, oxidative stress reduces the uptake of glutamate in astrocytes, a key step in the recycling of glutamate [16]. This potentially creates a vicious cycle leading to damage by free radicals unless the glutamatergic transmission is associated with increase in glutathione.

Other evidence implicates NMDA receptors in the interaction between glutathione and glutamate. In a recent review article, Hardingham and Do [17] presented evidence indicating that NMDAR hypofunction and oxidative stress may be reciprocally linked. For instance, glutathione enhances NMDAR responses whereas its depletion results in NMDAR hypofunction [18]. Furthermore, NMDAR hypofunction can also lead to oxidative damage [19]. Baxter et al. [20] demonstrated that synaptic activity, mediated via NMDA receptors, boosts the synthesis and utilization of glutathione, thereby adjusting antioxidant capacity to meet the elevated needs of active neurons. These studies indicate that in an equilibrium state there is likely to be a close coupling between glutamate and glutathione.

Magnetic resonance spectroscopy (MRS) allows biochemical concentrations of glutathione, glutamate, and other metabolites to be measured non-invasively in vivo. One MRS study found reduced glutathione in the medial prefrontal cortex (mPFC) in schizophrenia patients compared with healthy controls [21]. Another recent study reported reduced glutathione only in schizophrenia patients carrying a risk variant of the gene coding for the ratelimiting enzyme in glutathione synthesis [22]. Four other studies reported no statistically significant differences in glutathione levels in the posterior medial frontal cortex [23], mPFC [24] or anterior cingulate cortex (ACC) $[25,26]$. However, two out of the four found a trend toward reduced glutathione in schizophrenia $[24,25]$ and in one, lowered levels of glutathione were associated with negative symptoms [24]. On balance, these studies of predominantly wellestablished cases of schizophrenia indicate a tendency toward reduced glutathione. In contrast, a study of firstepisode cases reported elevated glutathione in medial temporal lobes [27] raising the possibility that glutathione abnormalities might vary with phase of illness. However, it should be noted that this study used a lenient threshold for the inclusion of glutathione metabolite fits.

Because glutamate released during neurotransmission is recycled via glutamine in glial cells, glutamine may index glutamate neurotransmission. However, glutamate and glutamine each participate in several other distinct cell processes and hence may not be closely coupled [28]. MRS studies of glutamate and glutamine concentrations in schizophrenia have been inconsistent, with some studies reporting an increase, others reporting a decrease or no abnormality ([29-35], for reviews see [36, 37]). One metaanalysis found that glutamate is reduced and glutamine is increased in the ACC in patients with schizophrenia, and both decrease more markedly with age in patients [38]. A 
more recent meta-analysis found higher medial-frontal Glx (glutamate + glutamine) levels in high-risk subjects [39]. Three recent studies used ultra-high field strength (7T) MRS, as its greater spectral resolution and signal-to-noise ratio help to distinguish between these two metabolites. Two studies investigated the $\mathrm{ACC} / \mathrm{mPFC}$ and one of these studies found increased glutamine/glutamate ratio [40] while the other found no significant abnormalities in glutamate levels in schizophrenia [41]. A third study found reduced glutamate in the occipital cortex in schizophrenia patients compared to healthy controls [42]. These studies provide some evidence of elevated glutamine levels in ACC/mPFC, especially in the early phase of illness. Overall, these studies indicate that glutamate may be unchanged or reduced, especially in more chronic cases, depending on the persistence of symptoms or other factors associated with long-term illness [29-37].

We reasoned that if decreases in glutathione and glutamate in the stable phase are a consequence of damage arising in a preceding acute phase, a sample of stable patients would be more likely to demonstrate a reduction in these neurochemicals than a more heterogeneous sample. We also predicted that any reduction in these neurochemicals would be most marked in cases of residual schizophrenia. ICD-10 defines residual schizophrenia as a chronic stage of the illness, in which there has been a progression from an early stage with positive psychotic symptoms to a later stage characterized by long-term negative symptoms and impairments, but reduced frequency and severity of positive symptoms [43]. Although the DSM-IV [44] does not explicitly define residual schizophrenia, it describes as typical a course of the illness in which positive symptoms diminish while negative symptoms persist. If residual schizophrenia reflects neural damage due to oxidative stress, reduced levels of glutamate and glutathione should be most marked in patients with residual schizophrenia.

As much of the MRS evidence in schizophrenia relates to the ACC, this was our primary region of interest. Insular cortex together with ACC forms the Salience Network, which is hypothesized to play an important role in schizophrenia [45]. Moreover, evidence indicates progressive structural changes in the insula early in the illness [46]. We therefore placed a voxel in the left insula. While much of the focus has been on the frontal cortex, it is possible that abnormalities in glutamatergic neurotransmission are widespread and include the visual cortex [47]. Hence, we chose the visual cortex as our third region of interest.

Since animal studies have demonstrated a close link between glutathione and glutamate uptake into both microglia and astrocytes, and NMDA receptor function, we also predicted that glutathione and glutamate levels in the brain would be correlated across all subjects. Although our primary hypothesis concerns glutathione and glutamate, the underlying hypothesis is that in the residual state there is diminished neural metabolic integrity arising from a toxic process in the acute phase of illness [4]. We might therefore predict that other metabolites would also exhibit reductions correlated with reductions in glutathione and glutamate. We therefore examined the correlations between glutathione, glutamate, and other metabolites reflecting cellular metabolic processes.

\section{Methods}

\section{Participants}

Patients aged 18-55 with a diagnosis of schizophrenia or schizoaffective disorder were referred to the study by community-based mental healthcare teams in Nottinghamshire, Derbyshire and Lincolnshire, England (for inclusion and exclusion criteria, see SA1 (Supplement).

All patients were in a stable phase of illness, defined as a change of no more than 10 points in their Social and Occupational Functioning Assessment Scale (SOFAS) score (defined in DSM-IV [44]) between assessment 6 weeks prior to and immediately prior to study participation. Patients with a documented history of prominent positive symptoms of schizophrenia but currently exhibiting no substantial positive symptoms were deemed to satisfy ICD-10 criteria for residual schizophrenia if they exhibited appreciable negative symptoms, and/or impaired occupational and/or social function. Operational criteria are specified formally in SA2.

Most patients were receiving psychotropic medication. The median defined daily dose (DDD) [48] was calculated separately for antipsychotics, mood stabilizers including lithium, and antidepressants. No patient had had a change in any of these medications 6 weeks prior to participating in this study.

Healthy participants with no personal or family history of psychotic disorders were recruited from the local community via posters, and matched group-wise to the patient group for age, gender, and parental socioeconomic status (SA3). Exclusion criteria were as for the patients.

All participants were assessed on scanning day for handedness, social and occupational functioning, and IQ. A clinical interview by a trained team member using a standardized symptom assessment was video-recorded (SA4).

This study was approved by the National Research Ethics Committee. All participants gave informed consent and received an inconvenience allowance. 

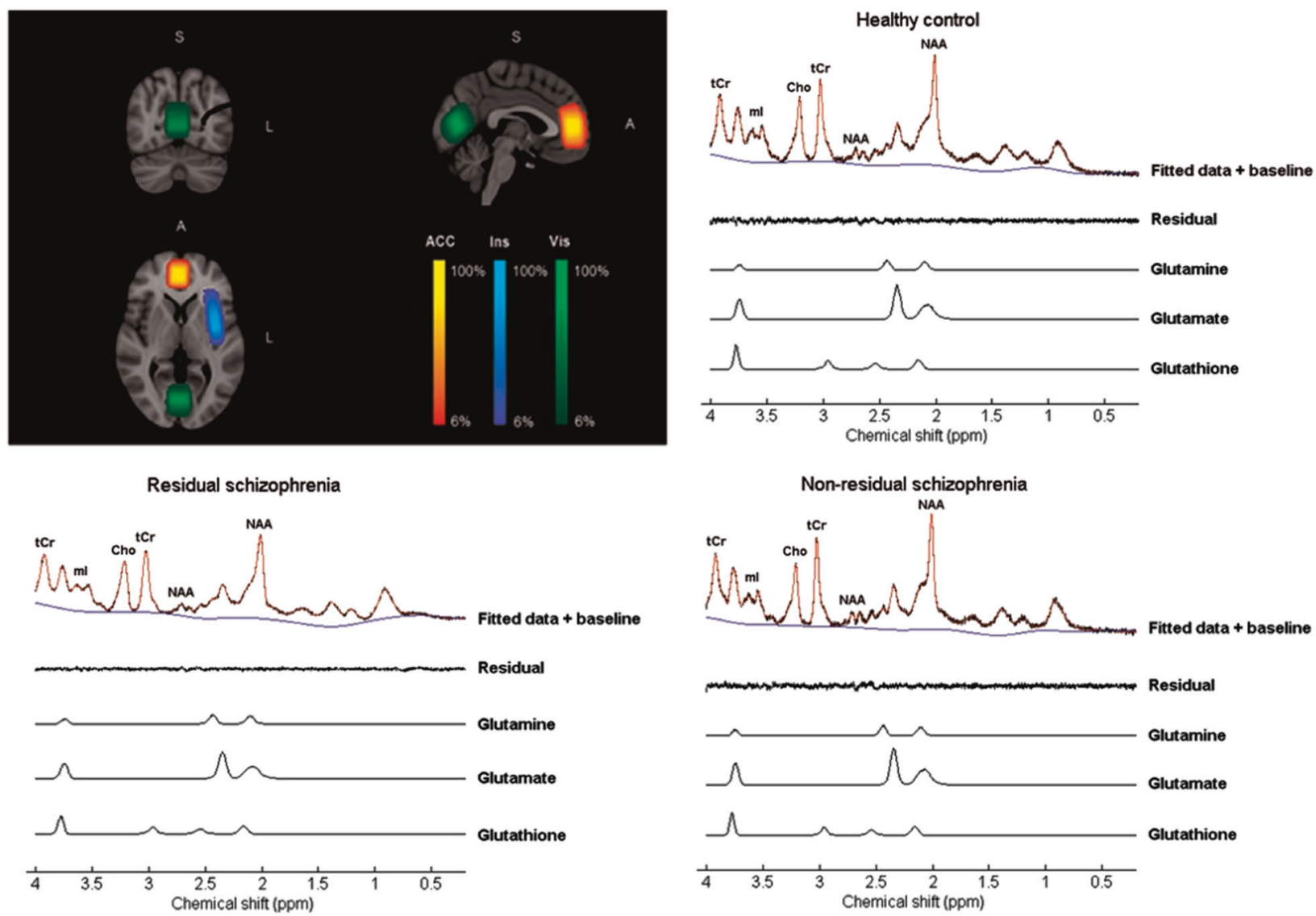

Fig. 1 Average voxel placements (ACC anterior cingulate cortex, Ins left insula, Vis visual cortex) across all subjects overlaid on an MNI brain; illustrative ${ }^{1} \mathrm{H}$ spectra with baseline and residuals from a voxel located in the ACC of subjects from all three groups (healthy controls,

residual schizophrenia, and non-residual schizophrenia) and corresponding fits from LCModel for glutamine (Gln), glutamate (Glu), and glutathione (GSH)

\section{Image acquisition}

Scans were conducted at the Sir Peter Mansfield Imaging Centre, University of Nottingham, using a 7 Tesla Philips Achieva scanner (Philips Medical Systems, Best, The Netherlands), a volume transmit head coil, and a 32-channel receive head coil. MRS data were collected using a ${ }^{1} \mathrm{H}$ MRS single voxel short TE STEAM (STimulated Echo Acquisition Mode) sequence (TE/TM/TR $=17 / 17 / 2000 \mathrm{~ms})$ with eight phase cycle steps, 4096 samples, and a $4 \mathrm{kHz}$ bandwidth. Two-hundred and eighty-eight spectra were collected using the Multiply Optimized Insensitive Suppression Train (MOIST) technique for water suppression. Two spectra were collected without water suppression in order to correct for absolute concentrations using water referencing. A $\mathrm{B}_{0}$ field map was acquired and parcellated shimming was used to enhance $B_{0}$ homogeneity [49]. The primary voxel of interest (VOI) $\left(20 \times 18 \times 25 \mathrm{~mm}^{3}\right)$ was placed in the ACC; a second VOI $\left(40 \times 12 \times 18 \mathrm{~mm}^{3}\right)$ was placed in the left insula; and third VOI $\left(20 \times 22 \times 20 \mathrm{~mm}^{3}\right)$ in the visual cortex. See Fig. 1 for a sample spectrum and voxel placements. An anatomical T1 MPRAGE

image $(\mathrm{TE} / \mathrm{TR}=3.4 / 7.3 \mathrm{~ms})$ was acquired for each subject $(1 \mathrm{~mm}$ isotropic resolution, $256 \times 256 \times 180$ matrix, flip angle $8^{\circ}$ ) to aid placement of the VOIs and for coregistration.

\section{Data processing}

For details of the procedure for estimating the metabolite concentrations in the three voxels see SA5. Briefly, the anatomical image was segmented into grey matter, white matter, and cerebrospinal fluid. MRS data acquired independently from the 32 channels were combined using an optimized coil combination method [50]. Metabolite concentrations were estimated using LCModel [51]. Data exceeding a threshold of $\mathrm{SD}=20 \%$ for the Cramer-Rao Lower Bound estimate of the precision of the quantification were excluded from the analysis. Metabolite concentrations were normalized to the concentration of water calculated from unsuppressed water spectra, since variations in water content within the sample are expected to be much smaller than the variations in the metabolite level [52]. Tissue volume fractions determined from the segmented images were used to correct metabolite 


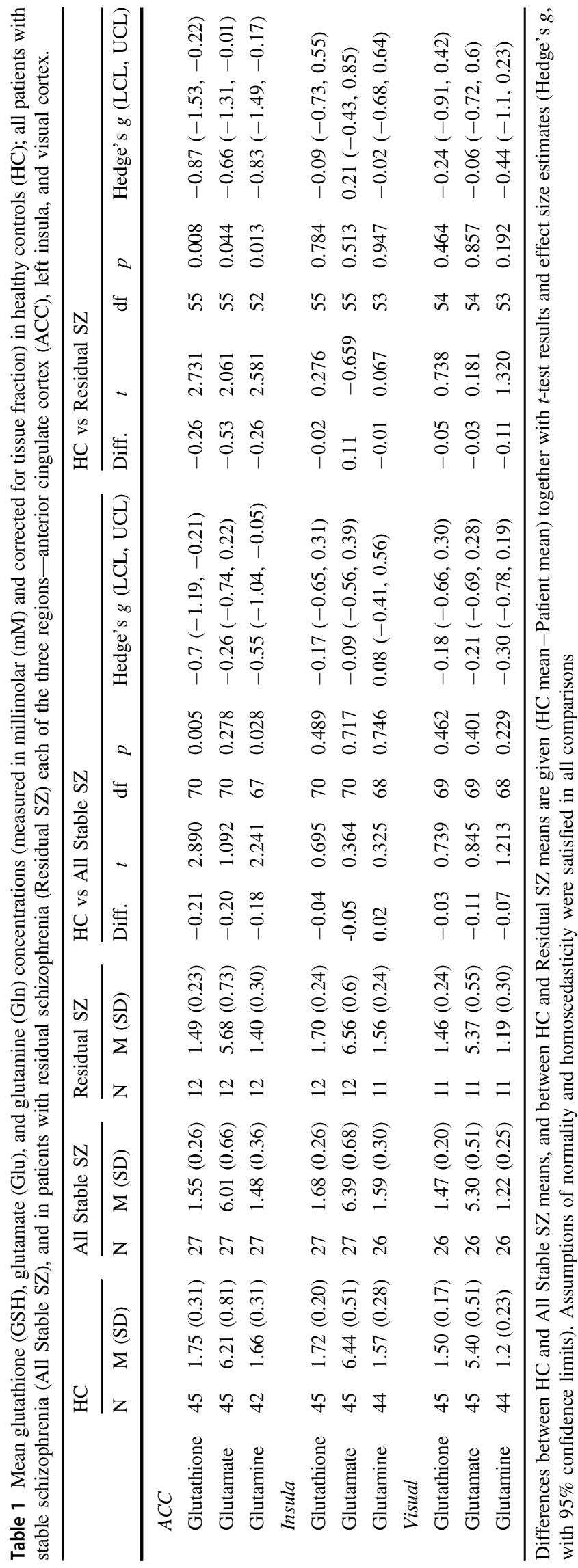

concentrations for partial volume effects and relaxation attenuation in each voxel [53]. Tissue composition and MRS data quality measures are reported in ST1-ST3.

\section{Statistical analyses}

As Pearson correlation coefficients between the nine metabolite concentration values (three metabolites in three voxels) were substantial even after adjustment for potential confounders, we used principal component analysis (PCA) to identify latent metabolite variables, and extracted scores for each component (see Results and SA7 for details). The PCA was weighted by group size; missing values were replaced by the mean. Statistical analyses were performed using SPSS 22 (IBM).

To determine, firstly, whether mean metabolite component scores in patients with stable schizophrenia differ from mean scores in healthy controls, and secondly, whether this difference is significantly leveraged by the subgroup of patients with residual schizophrenia, we regressed each component score in turn on two participant variables using a hierarchical regression model. In Block 1, we entered a binary dummy variable, "Stable Schizophrenia", coded 1 for a patient with schizophrenia and 0 otherwise. In Block 2, we entered an additional binary dummy variable, "Residual Schizophrenia", coded 1 for a patient meeting criteria for residual schizophrenia, and 0 otherwise.

To facilitate comparison with other studies, we also used independent samples $t$-tests to test for differences in metabolite concentrations between controls and (1) all patients, and (2) patients with residual schizophrenia. We also calculated Pearson correlations between glutathione, glutamate, glutamine, creatine, myo-inositol, and $n$-acetyl aspartate, and between metabolite PCA component scores and age; and antipsychotic DDD.

\section{Results}

\section{Participants}

Data from 45 healthy participants and 28 patients with schizophrenia were included in the analysis. Thirteen of the patients met criteria for residual schizophrenia (see SA6 for details of exclusions and a discussion relating to our sample size, clinical and demographic features of the sample are reported in ST4).

\section{LCModel results}

Means for glutathione, glutamate, and glutamine concentrations (estimated by LCModel and corrected for tissue 

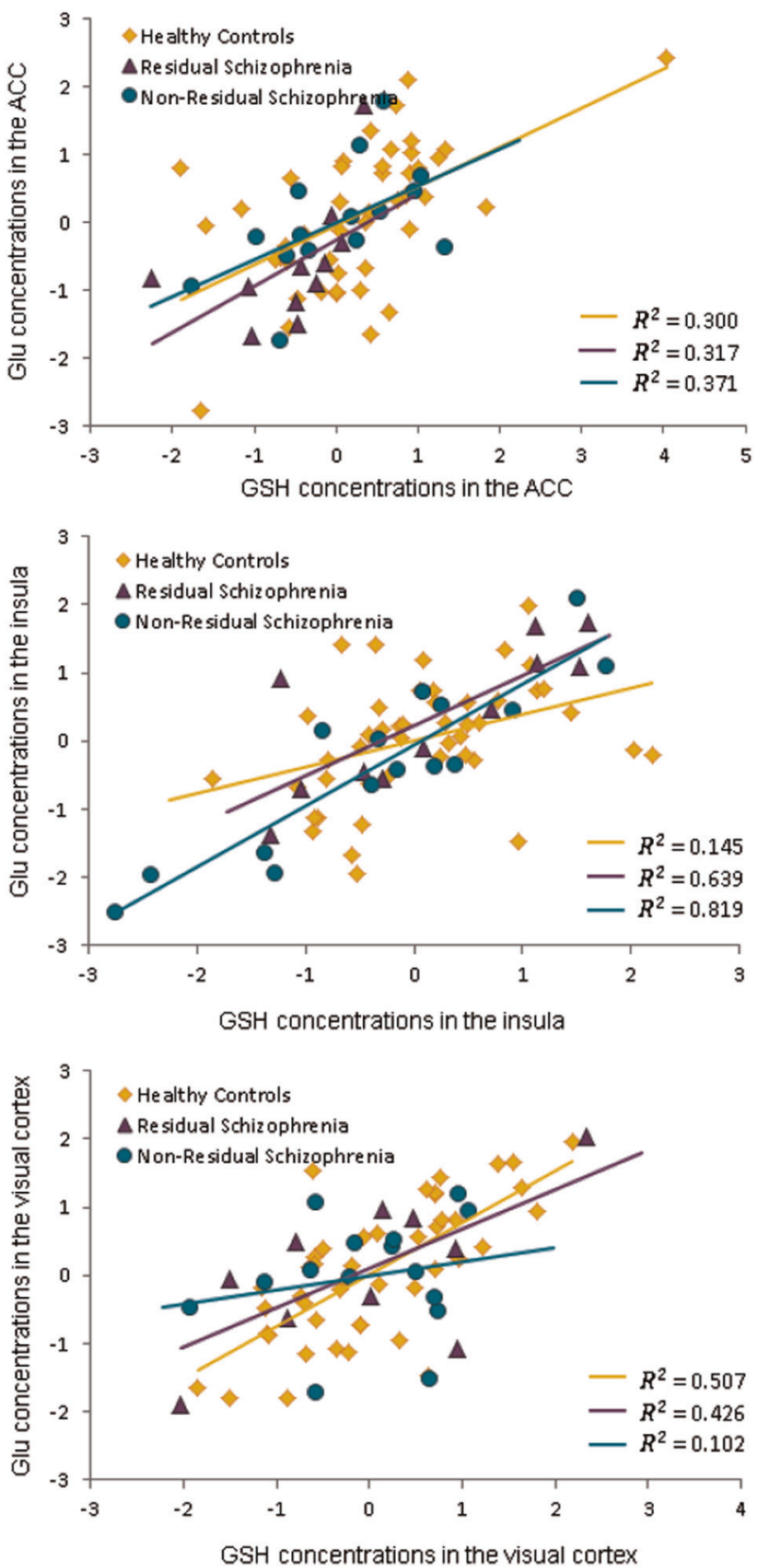

Fig. 2 Correlation between glutathione (GSH) and glutamate (Glu) concentrations in the three regions (anterior cingulate cortex (ACC), visual cortex, and insula) in the three groups (healthy controls, patients with residual schizophrenia, and patients with non-residual schizophrenia). Values represent standardized residuals after covarying for gender in the ACC and insula; and gender and spectral line-width in the visual cortex. Note: For the correlation between GSH and Glu in the ACC in healthy controls and in the insula in patients with residual schizophrenia, data did not satisfy the assumption of multivariate normality. Therefore, bootstrapped bias-corrected accelerated 95\% confidence intervals were computed (10,000 samples), and the correlations remained significant

fraction) in each of the three voxels are given in Table 1 for healthy controls, all patients with stable schizophrenia and in the sub-group of patients with residual schizophrenia (for other metabolites see ST5).
Table 2 Weighted principal components analysis (PCA) loadings for glutathione, glutamate, and glutamine in the whole sample. Bold font indicates loadings greater than 0.6 .

3 principal components explaining $64.82 \%$ of the variance (varimax rotation)

\begin{tabular}{lccc}
\hline Variables & \multicolumn{3}{l}{ Component loadings } \\
\cline { 2 - 4 } & Component 1 & Component 2 & Component 3 \\
\hline Ins glutathione & $\mathbf{0 . 7 4 6}$ & 0.254 & 0.116 \\
Ins glutamate & $\mathbf{0 . 8 0 8}$ & 0.184 & -0.059 \\
Vis glutathione & $\mathbf{0 . 7 3 8}$ & 0.028 & 0.013 \\
Vis glutamate & $\mathbf{0 . 8 3 5}$ & 0.070 & -0.096 \\
ACC glutathione & 0.160 & $\mathbf{0 . 8 7 1}$ & 0.010 \\
ACC glutamate & 0.210 & $\mathbf{0 . 8 0 4}$ & -0.080 \\
ACC glutamine & -0.070 & 0.382 & $\mathbf{0 . 7 2 5}$ \\
Ins glutamine & -0.004 & -0.076 & $\mathbf{0 . 7 5 9}$ \\
Vis glutamine & 0.026 & -0.168 & $\mathbf{0 . 6 9 7}$ \\
\hline
\end{tabular}

\section{Correlations and confounds}

As metabolite concentrations tended to be systematically higher in men than in women, we adjusted the values for gender (see SA7 for details). We found significant bivariate Pearson correlations between adjusted glutathione and glutamate in all three voxels $(p<0.05)$ (Fig. 2). Glutamine levels were significantly correlated across all three voxels, with $r$-values ranging from 0.28 to $0.36(p<0.05)$. The pattern of correlations between metabolites in the patient and control groups examined separately was similar to that in the combined group.

The PCA of the nine adjusted metabolite concentration values (glutathione, glutamate, and glutamine in each of the three voxels, ACC, Insula, and Visual) resulted in three components with an eigenvalue greater than 1. Component loadings after varimax rotation are given in Table 2 . The first component (accounting for $28 \%$ of the variance) loaded positively on glutathione and glutamate measures in the visual and insula voxels. The second component (18.7\% of the variance) loaded positively on glutathione and glutamate in the ACC and the third component (18\% of the variance) loaded positively on glutamine measures in all three voxels. Separate PCA in patient and control groups identified three similar components with the same metabolite loadings exceeding 0.5 on corresponding components in each group.

There were no significant correlations between metabolite component scores and antipsychotic dose in either patient group, nor were there significant correlations with age. Significant positive correlations were observed between several of the metabolites, specifically between glutathione, glutamate, NAA, creatine in the ACC (ST6). 
A

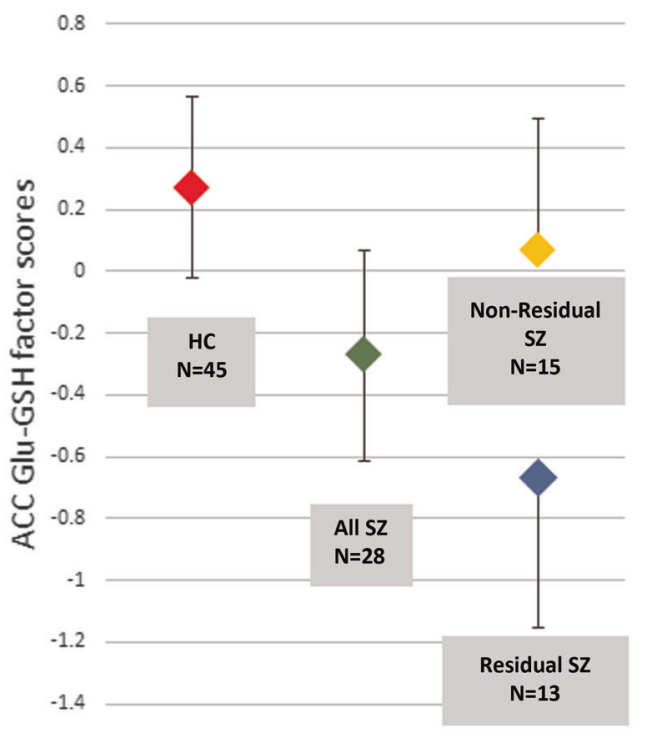

Fig. 3 Panel a shows mean component scores for the second PCA component loading on glutathione and glutamate concentrations in the ACC. Error bars represent $95 \%$ confidence intervals. There were no significant differences in variances between any groups or sub-groups. Panel b shows the effect sizes for differences in mean metabolite

\section{Hierarchical models}

Our primary hypothesis concerned the ACC, glutathione, and glutamate. The second PCA component, loading on glutathione and glutamate scores in the ACC, was therefore our first dependent variable. When "Stable Schizophrenia" was entered as a predictor, the $R^{2}$ of the model was $0.07, F$ $(1,71)=5.332, p=0.024$, a small effect size, $f^{2}=0.08$. The regression coefficient was significantly negative, indicating that mean component score was significantly lower in patients than in controls. When "Residual Schizophrenia" was included in the model, the $R^{2}$ of the model increased to 0.12 , a medium effect size, $f^{2}=0.14$. This $R^{2}$ increase was statistically significant, $F(23,70)=4.114, p=0.046$. The regression coefficient for "Residual Schizophrenia" was significantly negative, indicating that mean score in patients with residual schizophrenia was significantly lower than in either the other patients or than healthy controls. In this model, "Stable Schizophrenia" was no longer a significant predictor, indicating that patient-control difference in the first model was being leveraged almost entirely by the presence in the patient group of the patients with residual schizophrenia. These results are shown graphically in Fig. 3a.

For the first and third PCA components (glutathione and glutamate in the Insula and Visual voxels; glutamine in all three voxels), neither predictor accounted for

\section{B}

$-2.0$

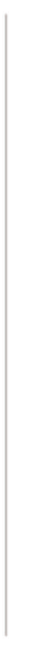

concentrations in the ACC between healthy controls and all patients (green bars) and the sub-group of patients with residual schizophrenia (blue bars). Error bars represent 95\% confidence limits of the effect size.

significant variance in component scores. Normality and homoscedasticity assumptions were satisfied in all models.

\section{Independent samples $t$-tests}

Results from independent samples $t$-tests on the nine unadjusted metabolite concentrations (glutathione, glutamate, and glutamine in each of the three voxels) are given in Table 1. Group mean metabolite concentrations were significantly lower in the patients than in the controls for both glutathione and glutamine in the ACC, and significantly lower for all three metabolites in the ACC in the subset of patients with residual schizophrenia. Effect sizes are plotted in Fig. 3b.

\section{Discussion}

Patients with stable schizophrenia had lower levels of glutathione and glutamate in the ACC, as measured by component scores representing variance shared between glutathione and glutamate in the ACC. This is consistent with our hypothesis that this group would include patients in whom excitotoxic damage during acute florid illness has led to subsequent reductions in mental and neural activity mediated by glutamatergic 
neurotransmission [3, 4]. Our hypothesis is further supported by the finding that for the sub-group of patients defined a priori as having residual schizophrenia, the effect size was larger, and indeed was largely responsible for the difference in scores between patients and controls. This finding is consistent with prior evidence indicating increased glutamatergic transmission early in the illness but diminished glutamate in older or more functionally impaired cases [29-37]. All three metabolites measured in the ACC were significantly lower in the group with residual schizophrenia than in the control group.

Glutathione and glutamate were significantly correlated with each other in all three brain regions. These high correlations are consistent with the hypothesis of a mechanistic link between antioxidant and glutamatergic systems in the human brain such that under steady-state conditions, low levels of glutathione are associated with low levels of glutamatergic neurotransmission [17]. In principle, the use of the unsuppressed water signal as a reference for metabolite signal normalization might introduce spurious correlations between glutathione and glutamate signals. However, provided variations in the water content within the sample are much smaller than the variations in the metabolite levels, variance shared between normalized metabolite signals can be assumed to arise largely from shared variance in metabolite concentrations [52]. It is noteworthy that there were no significant group differences in grey and white matter tissue composition in the three voxels (ST1).

Positive correlations exceeding an uncorrected threshold within each voxel were observed with all metabolites assessed apart for glutamine. After correction for multiple comparisons, correlations between glutathione, glutamate, NAA, creatine, and myo-inositol in the ACC remained significant. Component scores on a principal component representing variance shared between these five metabolites (ST7) were significantly reduced in residual schizophrenia compared with healthy controls. This suggests that these metabolites reflect metabolic integrity of cells (see SA8 for further discussion).

Ultra-high field MRS distinguishes between glutamate and glutamine, facilitating measures of glutathione and glutamate that are largely independent of glutamine levels. We found no significant correlations between glutamate and glutamine within any of the three voxels. However, we found significant correlations between glutamine levels across all three voxels, and all three glutamine measures loaded on a single component. This may be unsurprising, given the primary role of glutamine in glutamate recycling, a process likely to be cortex-wide.

Several limitations could be addressed in future studies. Firstly, given the evidence that glutathione and glutamate levels in the brain are affected by a risk variant of the gene coding for the glutathione synthesis enzyme, the role of genetic influences should be investigated [22]. Secondly, although we controlled for current antipsychotic medication intake and metabolite concentrations, possible confounding effects of long-term medication use should be considered. Thirdly, these results need to be interpreted with caution due to the potential differences in $\mathrm{T} 1$ and $\mathrm{T} 2$ relaxation times between different individuals and groups and their effects on neurochemical concentrations. Pragmatic constraints precluded measurement of water and metabolite $\mathrm{T} 1$ and $\mathrm{T} 2$ relaxation times in individual subjects. Instead, we used a short TE $(17 \mathrm{~ms})$ to minimize the effects of any potential differences in $\mathrm{T} 2$ relaxation times. While we cannot disregard the possibility of some remaining effects of relaxation time differentials, we note that our results are metabolite-specific, while systematic between-group differences in relaxation times would likely impact all metabolite concentrations equally.

Lastly, although the total number of patients was substantial, the cell size of the residual sub-group was small. Future studies with larger cell sizes should be conducted to compare sub-groups patients with different symptom profiles. Mostly importantly, our findings should motivate longitudinal studies using 7T MRS to follow neurochemical changes in individual patents across the phases of their illness.

\section{Disclaimer}

None of the funding bodies played any role in design and conduct of the study; collection, management, analysis, and interpretation of the data; and preparation, review, or approval of the manuscript; and decision to submit the manuscript for publication.

Acknowledgements This work was supported by the Medical Research Council [grant numbers MR/J01186X/1, G0901321, MR/ M006301/1], (the third grant is a UK New Investigator Research Grant supporting MJB); the Wellcome Trust [Research Training Fellowship WT096002/Z/11/Z] (supporting LP); the FP7 Marie Curie Actions of the European Commission [grant number FP7-PEOPLE-2012-ITN316716] (studentship supporting CF) and Mental Health Research UK (studentship supporting JK).

\section{Compliance with ethical standards}

Conflict of interest LP is an employee of Western University, Ontario. He receives book royalties from Oxford University Press and income from the SPMM MRCPsych course. In the last 2 years, his or his spousal pension funds held shares of Shire Inc., and GlaxoSmithKline. $\mathrm{He}$ has received travel support to speak at a meeting organized by Magstim Ltd (UK). The remaining authors declare that they have no conflict of interest. 
Open Access This article is licensed under a Creative Commons Attribution 4.0 International License, which permits use, sharing, adaptation, distribution and reproduction in any medium or format, as long as you give appropriate credit to the original author(s) and the source, provide a link to the Creative Commons license, and indicate if changes were made. The images or other third party material in this article are included in the article's Creative Commons license, unless indicated otherwise in a credit line to the material. If material is not included in the article's Creative Commons license and your intended use is not permitted by statutory regulation or exceeds the permitted use, you will need to obtain permission directly from the copyright holder. To view a copy of this license, visit http://creativecommons. org/licenses/by/4.0/.

\section{References}

1. Abi-Dargham A, Grace A. Dopamine and schizophrenia. In: Weinberger DR, Harrison P, editors. Schizophrenia. 3rd ed. Oxford: Wiley-Blackwell; 2011. p. 413-32.

2. Krystal JH, Moghaddam B. Contributions of glutamate and GABA systems to the neurobiology and treatment of schizophrenia. In: Weinberger DR, Harrison P, editors. Schizophrenia. 3rd ed. Oxford: Wiley-Blackwell; 2011. p. 433-61. .

3. Moghaddam B, Krystal JH. Capturing the angel in "angel dust": twenty years of translational neuroscience studies of NMDA receptor antagonists in animals and humans. Schizophr Bull. 2012;38:942-9.

4. Deutsch SI, Rosse RB, Schwartz BL, Mastropaolo J. A revised excitotoxic hypothesis of schizophrenia: therapeutic implications. Clin Neuropharmacol. 2001;24:43-9.

5. Hu W, MacDonald ML, Elswick DE, Sweet RA. The glutamate hypothesis of schizophrenia: evidence from human brain tissue studies. Ann NY Acad Sci. 2015;1338:38-57.

6. Shi J, Gershon ES, Liu C. Genetic associations with schizophrenia: meta-analyses of 12 candidate genes. Schizophr Res. 2008;104:96-107.

7. Stone JM. Glutamatergic antipsychotic drugs: a new dawn in the treatment of schizophrenia? Ther Adv Psychopharmacol. 2011;1:5-18.

8. Wood SJ,Yücel M,Pantelis C,Berk M, Neurobiology of schizophrenia spectrum disorders: the role of oxidative stress. Ann Acad Med Singapore. 2009;38:396

9. Raffa M, Mechri A, Othman LB, Fendri C, Gaha L, Kerkeni A. Decreased glutathione levels and antioxidant enzyme activities in untreated and treated schizophrenic patients. Prog Neuropsychopharmacol Biol Psychiatry. 2009;33:1178-83.

10. Yao JK, Leonard S, Reddy R. Altered glutathione redox state in schizophrenia. Dis Markers. 2006;22:83-93.

11. Gawryluk JW, Wang JF, Andreazza AC, Shao L, Young LT. Decreased levels of glutathione, the major brain antioxidant, in post-mortem prefrontal cortex from patients with psychiatric disorders. Int J Neuropsychopharmacol. 2011;14: 123-30.

12. Gysin R, Kraftsik R, Sandell J, Bovet P, Chappuis C, Conus P, et al. Impaired glutathione synthesis in schizophrenia: convergent genetic and functional evidence. Proc Natl Acad Sci USA. 2007;104:16621-6.

13. Persson M, Sandberg M, Hansson E, Rönnbäck L. Microglial glutamate uptake is coupled to glutathione synthesis and glutamate release. Eur J Neurosci. 2006;24:1063-70.

14. Barger SW, Goodwin ME, Porter MM, Beggs ML. Glutamate release from activated microglia requires the oxidative burst and lipid peroxidation. J Neurochem. 2007;101:1205-13.
15. Bains JS, Shaw CA. Neurodegenerative disorders in humans: the role of glutathione in oxidative stress-mediated neuronal death. Brain Res Brain Res Rev. 1997;25:335-58.

16. Volterra A, Trotti D, Tromba C, Floridi S, Racagni G. Glutamate uptake inhibition by oxygen free radicals in rat cortical astrocytes. J Neurosci. 1994;14:2924-32.

17. Hardingham GE, Do KQ. Linking early-life NMDAR hypofunction and oxidative stress in schizophrenia pathogenesis. Nat Rev Neurosci. 2016;17:125-34.

18. Steullet P, Neijt HC, Cuénod M, Do KQ. Synaptic plasticity impairment and hypofunction of NMDA receptors induced by glutathione deficit: relevance to schizophrenia. Neuroscience. 2006;137:807-19.

19. Papadia S, Soriano FX, Léveillé F, Martel MA, Dakin KA, Hansen $\mathrm{HH}$, et al. Synaptic NMDA receptor activity boosts intrinsic antioxidant defences. Nat Neurosci. 2008;11:476-87.

20. Baxter PS, Bell KF, Hasel P, Kaindl AM, Fricker M, Thomson D, et al. Synaptic NMDA receptor activity is coupled to the transcriptional control of the glutathione system. Nat Commun. 2015;6:6761.

21. Do KQ, Trabesinger AH, Kirsten-Krüger M, Lauer CJ, Dydak U, Hell D, et al. Schizophrenia: glutathione deficit in cerebrospinal fluid and prefrontal cortex in vivo. Eur $\mathbf{J}$ Neurosci. 2000;12:3721-8.

22. Xin L, Mekle R, Fournier M, Baumann PS, Ferrari C, Alameda L, et al. Genetic polymorphism associated prefrontal glutathione and its coupling with brain glutamate and peripheral redox status in early psychosis. Schizophr Bull. 2016;42:1185-96.

23. Monin A, Baumann PS, Griffa A, Xin L, Mekle R, Fournier M, et al. Glutathione deficit impairs myelin maturation: relevance for white matter integrity in schizophrenia patients. Mol Psychiatry. 2015;20:827-38.

24. Matsuzawa D, Obata T, Shirayama Y, Nonaka H, Kanazawa Y, Yoshitome E, et al. Negative correlation between brain glutathione level and negative symptoms in schizophrenia: a $3 \mathrm{~T} 1 \mathrm{H}-$ MRS study. PLoS ONE. 2008;3:e1944.

25. Terpstra M, Vaughan TJ, Ugurbil K, Lim KO, Schulz SC, Gruetter R. Validation of glutathione quantitation from STEAM spectra against edited 1H NMR spectroscopy at 4T: application to schizophrenia. MAGMA. 2005;18:276-82.

26. Brandt AS, Unschuld PG, Pradhan S, Lim IA, Churchill G, Harris $\mathrm{AD}$, et al. Age-related changes in anterior cingulate cortex glutamate in schizophrenia: a ${ }^{1} \mathrm{H}$ MRS Study at 7 Tesla. Schizophr Res. 2016;172:101-5.

27. Wood SJ, Berger GE, Wellard RM, Proffitt TM, McConchie M, Berk M, et al. Medial temporal lobe glutathione concentration in first episode psychosis: a 1H-MRS investigation. Neurobiol Dis. 2009;33:354-7.

28. McKenna MC. The glutamate-glutamine cycle is not stoichiometric: fates of glutamate in brain. J Neurosci Res. 2007;85:3347-58

29. Marsman A, van den Heuvel MP, Klomp DW, Kahn RS, Luijten PR, Hulshoff Pol HE. Glutamate in schizophrenia: a focused review and meta-analysis of ${ }^{1} \mathrm{H}-\mathrm{MRS}$ studies. Schizophr Bull. 2013;39:120-9.

30. Merritt K, Egerton A, Kempton MJ, Taylor MJ, McGuire PK. Nature of glutamate alterations in schizophrenia: a meta-analysis of proton magnetic resonance spectroscopy studies. JAMA Psychiatry. 2016;73:665-74.

31. Rowland LM, Pradhan S, Korenic S, Wijtenburg SA, Hong LE, Edden RA, et al. Elevated brain lactate in schizophrenia: a 7T Magnetic Resonance Spectroscopy Study. Transl Psychiatry. 2016;6:e967.

32. Marsman A, Mandl RC, Klomp DW, Bohlken MM, Boer VO, Andreychenko A, et al. GABA and glutamate in schizophrenia: a 7T ${ }^{1}$ H-MRS Study. Neuroimage Clin. 2014;6:398-407. 
33. Thakkar KN, Rösler L, Wijnen JP, Boer VO, Klomp DW, Cahn $\mathrm{W}$, et al. 7T proton magnetic resonance spectroscopy of gammaaminobutyric acid, glutamate, and glutamine reveals altered concentrations in patients with schizophrenia and healthy siblings. Biol Psychiatry. 2017;81:525-35.

34. Bustillo JR, Chen H, Jones T, Lemke N, Abbott C, Qualls C, et al. Increased glutamine in patients undergoing long-term treatment for schizophrenia: a proton magnetic resonance spectroscopy study at 3T. JAMA Psychiatry. 2014;71:265-72.

35. Plitman E, de la Fuente-Sandoval C, Reyes-Madrigal F, Chavez S, Gómez-Cruz G, León-Ortiz P, et al. Elevated myo-inositol, choline, and glutamate levels in the associative striatum of antipsychotic-naivepatients with first-episode psychosis: a proton magnetic resonance spectroscopy study with implications for glial dysfunction. Schizophr Bull. 2016;42: 415-24.

36. Tayoshi SY, Sumitani S, Taniguchi K, Shibuya-Tayoshi S, Numata S, Iga J, et al. Metabolite changes and gender differences in schizophrenia using 3-Tesla proton magnetic resonance spectroscopy (1H-MRS). Schizophr Res. 2009;108:69-77.

37. Théberge J, Al-Semaan Y, Jensen JE, Williamson PC, Neufeld RW, Menon RS, et al. Comparative study of proton and phosphorus magnetic resonance spectroscopy in schizophrenia at 4 Tesla. Psychiatry Res. 2004;132:33-9.

38. Bustillo JR, Chen H, Gasparovic C, Mullins P, Caprihan A, Qualls $\mathrm{C}$, et al. Glutamate as a marker of cognitive function in schizophrenia: a proton spectroscopic imaging study at 4 Tesla. Biol Psychiatry. 2011;69:19-27.

39. Kraguljac NV, Reid MA, White DM, den Hollander J, Lahti AC. Regional decoupling of $\mathrm{N}$-acetyl-aspartate and glutamate in schizophrenia. Neuropsychopharmacology. 2012;37: 2635-42.

40. Egerton A, Brugger S, Raffin M, Barker GJ, Lythgoe DJ, McGuire PK, et al. Anterior cingulate glutamate levels related to clinical status following treatment in first-episode schizophrenia. Neuropsychopharmacology. 2012;37:2515-21.

41. Poels EM, Kegeles LS, Kantrowitz JT, Javitt DC, Lieberman JA, Abi-Dargham A, et al. Glutamatergic abnormalities in schizophrenia: a review of proton MRS findings. Schizophr Res. 2014;152:325-32.
42. Wijtenburg SA, Yang S, Fischer BA, Rowland LM. In vivo assessment of neurotransmitters and modulators with magnetic resonance spectroscopy: application to schizophrenia. Neurosci Biobehav Rev. 2015;51:276-95.

43. World Health Organization. ICD-10 classifications of mental and behavioural disorder: clinical descriptions and diagnostic guidelines. Geneva: World Health Organization; 1992.

44. American Psychiatric Association. Diagnostic and statistical manual of mental disorders: DSM-IV. 4th ed. Washington, DC: American Psychiatric Association; 1994.

45. Palaniyappan L, Liddle PF. Does the salience network play a cardinal role in psychosis? An emerging hypothesis of insular dysfunction. J Psychiatry Neurosci. 2012;37:17-27.

46. Palaniyappan L, Crow TJ, Hough M, Voets NL, Liddle PF, James $\mathrm{S}$, et al. Gyrification of Broca's region is anomalously lateralized at onset of schizophrenia in adolescence and regresses at 2year follow-up. Schizophr Res. 2013;147:39-45.

47. Javitt DC, Zukin SR, Heresco-Levy U, Umbricht D. Has an angel shown the way? Etiological and therapeutic implications of the PCP/NMDA model of schizophrenia. Schizophr Bull. 2012;38:958-66.

48. WHO Collaborating Centre for Drug Statistics and Methodology. Guidelines for ATC classification and DDD assignment. Oslo, Norway: WHO Collaborating Centre for Drug Statistics and Methodology; 2003.

49. Poole M, Bowtell R. Volume parcellation for improved dynamic shimming. MAGMA. 2008;21:31-40.

50. Hall EL, Stephenson MC, Price D, Morris PG. Methodology for improved detection of low concentration metabolites in MRS: optimised combination of signals from multi-element coil arrays. Neuroimage. 2014;86:35-42.

51. Provencher SW. Estimation of metabolite concentrations from localized in vivo proton NMR spectra. Magn Reson Med. 1993;30:672-9.

52. Neeb H, Ermer V, Stocker T, Shah NJ. Fast quantitative mapping of absolute water content with full brain coverage. Neuroimage. 2008;42:1094-109.

53. Gasparovic C, Song T, Devier D, Bockholt HJ, Caprihan A, Mullins PG, et al. Use of tissue water as a concentration reference for proton spectroscopic imaging. Magn Reson Med. 2006;55:1219-26. 